\title{
Consistent metric combinations in cosmology of massive bigravity
}

\author{
Henrik Nersisyan, ${ }^{1, *}$ Yashar Akrami, ${ }^{1, \oplus}$ and Luca Amendola ${ }^{1, \text {, }}$ \\ ${ }^{1}$ Institut Für Theoretische Physik, Ruprecht-Karls-Universität Heidelberg, Philosophenweg 16, 69120 Heidelberg, Germany
}

\begin{abstract}
Massive bigravity models are interesting alternatives to standard cosmology. In most cases, however, these models have been studied for a simplified scenario in which both metrics take homogeneous and isotropic forms [Friedmann-Lemaitre-Robertson-Walker (FLRW)] with the same spatial curvatures. The interest to consider more general geometries arises, in particular, in view of the difficulty so far encountered in building stable cosmological solutions with homogeneous and isotropic metrics. Here we consider a number of cases in which the two metrics take more general forms, namely FLRW with different spatial curvatures-Lemaître, Lemaître-Tolman-Bondi (LTB), and Bianchi I - as well as cases where only one metric is linearly perturbed. We discuss possible consistent combinations and find that only some special cases of FLRW-Lemaitre, LTB-LTB, and FLRW-Bianchi I combinations give consistent, nontrivial solutions.
\end{abstract}

Keywords: modified gravity, massive gravity, bimetric gravity, background cosmology

\section{INTRODUCTION}

The standard $\Lambda$ CDM model of cosmology is based on four main assumptions: general relativity (GR) is the correct description of gravitational interactions at energies below the Planck scale, the Universe is homogenous and isotropic on large scales (the cosmological principle), the energy content of the Universe is mainly in the form of cold dark matter (CDM) and a nondynamical cosmological constant $\Lambda$, and all the structure that we see around us originated from nearly Gaussian, adiabatic, and scale-independent quantum fluctuations at early times. All these assumptions have been tested with high precision using various cosmological data and seem to be in excellent agreement with all existing observations. There are, however, various theoretical reasons why one may want to go beyond this standard framework. In particular, the assumption that the late-time acceleration of the Universe is due to a cosmological constant term has been strongly questioned from the theoretical point of view, as its small but nonzero value preferred by observations cannot be explained by fundamental physics [1]. It is, therefore, important and quite natural to ask whether the cosmic acceleration can be explained by a different mechanism than a pure cosmological constant. One particular possibility, which has attracted remarkable attention over the last decade, is that a modification of GR on very large scales might be responsible for the acceleration (see Refs. [2, 3] for comprehensive reviews). One of the interesting such infrared modifications is to assume that gravitons are not massless as opposed to what GR tells us. A nonzero but sufficiently small graviton mass modifies properties of the gravitational interactions on very large scales while leaving them indistinguishable from the predictions of standard gravity on small scales where GR is believed to be at work.

GR is a consistent and nonlinear theory of massless gravity and, therefore, has given the possibility of constructing various cosmological models. In order to test the implications of massive gravity for cosmology, one similarly needs a nonlinear and consistent theory for massive gravitons. Such a theory was, however, not available for more than 70 years after the construction of a linear theory of massive gravity by Fierz and Pauli in 1939 [4]. This was mainly because any attempts at constructing a nonlinear completion of the Fierz and Pauli theory would face a serious obstacle; the theory would suffer from the existence of the so-called Boulware-Deser (BD) ghost degrees of freedom [5], a property which would be fatal to the theory. It was only a few years ago that a ghost-free and fully nonlinear formulation of massive gravity, and its bimetric extension, was constructed [6 14] (see Ref. [15] for a recent review). The key step for this success was to extend the gravitational sector by at least one new spin-2 tensor field with metric-like properties. In addition, in order to avoid the BD ghost, the physical metric of the theory has to interact with the new tensor field in a very specific way. In the simplest version of the theory, referred to as the de Rham-Gabadadze-Tolley (dRGT) theory of massive gravity, only the physical metric, the one which interacts with the matter sector in the standard way, is dynamical, i.e. has an Einstein-Hilbert term in the action, while the second metric, often called "reference" metric, does not have dynamics. In this case gravitons posses five degrees of freedom. In the bimetric version of the theory, referred to as the Hassan-Rosen theory of bigravity, the reference metric is also given dynamics and, therefore, gravitons posses seven degrees of freedom, corresponding to one massless and one massive graviton.

* $†$ h.nersisyan@thphys.uni-heidelberg.de

$\ddagger$ l.amendola@thphys.uni-heidelberg.de 
The dRGT theory of massive gravity has been shown to suffer from a no-go theorem forbidding flat and closed Friedmann-Lemaître-Robertson-Walker (FLRW) cosmological solutions on a flat reference metric [16]. In addition, dRGT with an open FLRW metric or a nonflat reference metric suffers from the so-called Higuchi instability [17] or other types of instabilities 18 23]. One obvious way to avoid the no-go theorem is to give up on exact FLRW solutions, i.e. to consider inhomogeneous and/or anisotropic solutions for the metrics. We, however, know that the observable Universe on large scales is very close to being homogenous and isotropic and, therefore, non-FLRW solutions, if allowed, must not deviate significantly from the FLRW case on observable scales, and must respect the observational bounds on inhomogeneity and anisotropy. Non-FLRW effects should be either of very low amplitudes or of scales much larger than our horizon so that they cannot be observed. Interestingly, solutions which satisfy these conditions have been shown to exist in dRGT [16]. Other scenarios with non-FLRW solutions in dRGT, either for the physical metric or for the reference metric, can be found in Refs. 21, 23 30] (see also Ref. [15] for a thorough review of the inhomogeneous and anisotropic solutions in massive gravity). The other possibility to avoid the no-go theorem and instability issues in dRGT is to extend the theory. An example for such extensions without adding new degrees of freedom is the recently proposed generalized massive gravity theory [31]. Another workaround is through theories with extra degrees of freedom or violation of certain symmetries; these include for example quasidilaton [32], varying-mass [16, 33], nonlocal [34 37], and Lorentz-violating [38, 39] massive gravity. Recently, another solution to the no-go theorem has been proposed in Refs. [40 42]. It has been suggested that the no-go theorem can be overcome if at least some matter couples to a hybrid metric, composed of both the physical and reference metrics and constructed in a specific way to keep the theory free of the BD ghost up to a cut-off energy scale which is believed to be above the strong coupling scale of the theory. This makes phenomenological studies of the theory possible below the cut-off scale (see, however, Ref. 43] for various complications that the cosmology of this theory would need to tackle). The revival of the BD ghost is a general feature of the scenarios where both metrics couple simultaneously to matter [40, 41, 44 48]; these include the simplest case where the two metrics couple to matter minimally [49 52].

The Hassan-Rosen theory of massive bigravity on the other hand is immune from the no-go theorem and admits usual flat FLRW solutions. The cosmology of bigravity has been extensively studied in the literature at both the background and perturbative levels (see, e.g., Refs. [53 71]) and in terms of the lensing and dynamical properties of local sources [61] (see also Refs. [72 75] for cosmological studies of bigravity where matter couples to both metrics through a composite metric). It has particularly been shown that the theory can explain the late-time acceleration of the Universe in the absence of an explicit cosmological constant and can, therefore, serve as a viable alternative to $\Lambda$ CDM [59, 63] at the background level. However, when it comes to perturbations, the Hassan-Rosen theory of massive bigravity seems to be suffering from various instabilities. Bigravity models can generally be classified into two categories, finite and infinite branches. This classification is based on the fact that the ratio of the two scale factors for the reference and physical metrics in FLRW solutions is either increasing (finite branch) or decreasing (infinite branch) with time, depending on which combinations of the parameters of the theory are nonvanishing. It has been shown that scalar perturbations for all finite-branch models, including a simple single-parameter model called minimal bigravity model (MBM) [64], are unstable at early times on small scales [56, 64, 66, 68, 69], although the models are viable at the background level. The instabilities do not necessarily rule these models out but make their comparison to observations difficult as one can no longer employ linear perturbation theory to study their implications for the formation of structure. Linear scalar perturbations for the infinite-branch bigravity (IBB), as identified in Ref. [68], are on the other hand stable at all times, making the study of structure formation possible for the model. This was done in Refs. 67, 68], where subhorizon scales were analyzed in the quasistatic limit, various modified gravity parameters were calculated, and deviations from GR predictions were presented. It was shown that predictions of the model are consistent with existing large-scale structure data and the model can be tested by future experiments. IBB was, however, shown later to suffer from two other types of instability; it violates the Higuchi bound [69, 76], which can potentially be dangerous as the instabilities might appear at higher-order perturbations for nonlinear structure, and tensor perturbations have ghost instabilities at early times [70, 76 78]. Addressing the problem of instabilities in bigravity is currently an active field of research. In principle most of the generalizations of the dRGT theory of massive gravity enumerated in the previous paragraph can be applied to bigravity to investigate possible resolutions to the instability problems and to construct viable alternatives to $\Lambda$ CDM.

Motivated by these instability problems in the standard scenario of massive bigravity with FLRW metrics, in this paper we take the first step in exploring one potential route to resolve the obstacles and construct a viable and stable model. It is not clear at this stage whether possible solutions are necessarily in modifications of the structure of the theory, i.e. at the level of the action. Such possibilities should definitely be explored, but one should also consider cases where the structure of the theory remains intact while other classes of solutions are considered. One of these possibilities is the class of solutions with non-FLRW metrics for one or both metrics of the theory. As we mentioned earlier, this has been shown to be a promising route in the case of dRGT, and it is, therefore, worth investigating for bigravity as well. Before studying the cosmological implications of such cases, one needs to check whether solutions to field equations exist and whether they are consistent with basic constraints of the theory, such as Bianchi constraints. 
This is the objective of the present paper.

Inhomogeneous and anisotropic solutions in bigravity have been studied in the literature. This includes Bianchi cosmologies where both metrics are homogeneous and anisotropic [30, 79], as well as cosmologies with the physical metric being FLRW and the reference metric being inhomogeneous [26]. In this paper we study various combinations of FLRW and non-FLRW metrics in a systematic and more general way, and investigate for which combinations consistent solutions to the equations of motion exist. Our aim is to identify such combinations without further exploration of their implications for cosmology; we leave this for future work.

The rest of this paper is organized as follows. In Sec. II we review the Hassan-Rosen theory of singly-coupled bigravity and present the field equations and Bianchi constraints. In Sec. III we study various combinations of FLRW, inhomogeneous, and anisotropic solutions for the metrics of the theory. We start this in Sec. IIIA with the case where both metrics are of the FLRW form but have different spatial curvatures. Since, as we will see, this will force the metrics to be of the Lemaitre form, we study those solutions in the same section. We move on in Sec. IIIB with the combinations where one metric is FLRW and the other one is of the Lemaitre-Tolman-Bondi (LTB) form, and in Sec. IIIC we investigate the solutions where both metrics are LTB. Combinations of FLRW and anisotropic but homogeneous metrics are studied in Sec. IIIE in the context of Bianchi type I solutions. In all these sections we discuss the consistency of the solutions when matter sources respect or violate the homogeneity or anisotropy assumptions. In Sec. IV we go beyond the background solutions and investigate the scenarios where one metric is perturbed while the other one is kept unperturbed. Our discussions are based on both scalar and tensor perturbations. We discuss our results and conclude in Sec. V.

\section{THE THEORY OF MASSIVE BIGRAVITY}

The Hassan-Rosen theory of ghost-free, massive bigravity is characterized by the action [13]

$$
\begin{aligned}
S & =-\frac{M_{g}^{2}}{2} \int d^{4} x \sqrt{-\operatorname{det} g} R_{g}-\frac{M_{f}^{2}}{2} \int d^{4} x \sqrt{-\operatorname{det} f} R_{f} \\
& +m^{4} \int d^{4} x \sqrt{-\operatorname{det} g} \sum_{n=0}^{4} \beta_{n} e_{n}\left(\sqrt{g^{-1} f}\right)+\int d^{4} x \sqrt{-\operatorname{det} g} \mathcal{L}_{m}(g, \Phi),
\end{aligned}
$$

where $M_{g}$ and $M_{f}$ are Planck masses and $R_{g}$ and $R_{f}$ are the Ricci scalars for the metrics $g_{\mu \nu}$ and $f_{\mu \nu}$, respectively. Here $g_{\mu \nu}$ is the standard, physical metric coupled to matter fields $\Phi$ through the matter Lagrangian $\mathcal{L}_{m}$, and $f_{\mu \nu}$ is the reference metric. The action contains five interaction (mass) terms given in terms of five functions $e_{n}$. These are the elementary symmetric polynomials of the eigenvalues of the matrix $\sqrt{g^{-1} f}$, where $\sqrt{g^{-1} f} \sqrt{g^{-1} f} \equiv g^{\mu \nu} f_{\mu \nu}$. The forms of these polynomials are presented in, e.g., Ref. [13]. The quantities $\beta_{n}(n=0,1,2,3,4)$ are free parameters of the theory, and $m$ is the mass parameter. In the following we express masses in units of $M_{g}^{2}$ and absorb $m^{4}$ into the parameters $\beta_{n}$ ( $m$ is not an independent parameter of the theory). The action then becomes

$$
\begin{aligned}
S & =-\frac{1}{2} \int d^{4} x \sqrt{-\operatorname{det} g} R_{g}-\frac{M_{f}^{2}}{2} \int d^{4} x \sqrt{-\operatorname{det} f} R_{f} \\
& +\int d^{4} x \sqrt{-\operatorname{det} g} \sum_{n=0}^{4} \beta_{n} e_{n}\left(\sqrt{g^{-1} f}\right)+\int d^{4} x \sqrt{-\operatorname{det} g} \mathcal{L}_{m}(g, \Phi) .
\end{aligned}
$$

By varying the action (2) with respect to $g_{\mu \nu}$ one obtains the generalized Einstein equation for the physical metric,

$$
R_{\mu \nu}^{g}-\frac{1}{2} g_{\mu \nu} R_{g}+\sum_{n=0}^{3}(-1)^{n} \beta_{n} g_{\mu \lambda} Y_{(n) \nu}^{\lambda}\left(\sqrt{g^{-1} f}\right)=T_{\mu \nu},
$$

where $R_{\mu \nu}^{g}$ is the $g$-metric Ricci tensor, and the matrices $Y_{(n)}(X)$ are defined as $[13$.

$$
\begin{aligned}
Y_{(0)}(X) & \equiv I, \\
Y_{(1)}(X) & \equiv X-I[X], \\
Y_{(2)}(X) & \equiv X^{2}-X[X]+\frac{1}{2} I\left([X]^{2}-\left[X^{2}\right]\right), \\
Y_{(3)}(X) & \equiv X^{3}-X^{2}[X]+\frac{1}{2} X\left([X]^{2}-\left[X^{2}\right]\right)-\frac{1}{6} I\left([X]^{3}-3[X]\left[X^{2}\right]+2\left[X^{3}\right]\right),
\end{aligned}
$$


where $X \equiv\left(\sqrt{g^{-1} f}\right), I$ is the identity matrix, and $[\ldots]$ is the trace operator.

By varying the action (2) with respect to the reference metric $f_{\mu \nu}$ we obtain

$$
R_{\mu \nu}^{f}-\frac{1}{2} f_{\mu \nu} R_{f}+\frac{1}{M_{f}^{2}} \sum_{n=0}^{3}(-1)^{n} \beta_{4-n} f_{\mu \lambda} Y_{(n) \nu}^{\lambda}\left(\sqrt{g^{-1} f}\right)=0,
$$

where $R_{\mu \nu}^{f}$ is the $f$-metric Ricci tensor. Under the rescaling $f_{\mu \nu} \rightarrow M_{f}^{-2} f_{\mu \nu}$, the Ricci scalar $R_{f}$ transforms as $R_{f} \rightarrow M_{f}^{2} R_{f}$, which results in

$$
\sqrt{-\operatorname{det} f} R_{f} \rightarrow M_{f}^{-2} \sqrt{-\operatorname{det} f} R_{f} .
$$

The interaction terms in the action then transform as

$$
\sum_{n=0}^{4} \beta_{n} e_{n}\left(\sqrt{g^{-1} f}\right) \rightarrow \sum_{n=0}^{4} \beta_{n} e_{n}\left(M_{f}^{-1} \sqrt{g^{-1} f}\right) .
$$

Since the elementary symmetric polynomials $e_{n}(X)$ are of order $X^{n}$, the rescaling of $f_{\mu \nu}$ by a constant factor $M_{f}^{-2}$ translates into a redefinition of the coupling constants $\beta_{n} \rightarrow M_{f}^{n} \beta_{n}$, which allows us to assume $\left.M_{f}=1\right]$

In addition to the equations of motion for the metrics, there are additional constraints on the dynamics of the metrics coming from the Bianchi identities and the assumption that the stress-energy-momentum tensor of the matter components is conserved,

$$
\frac{1}{2} \nabla_{g}^{\mu} \sum_{n=0}^{3}(-1)^{n} \beta_{n} g_{\mu \lambda} Y_{(n) \nu}^{\lambda}\left(\sqrt{g^{-1} f}\right)=0,
$$

where $\nabla_{g}$ is the covariant derivative operator with respect to $g_{\mu \nu}$. Any acceptable bigravity solution must satisfy the generalized Einstein equations (3) and (4), as well as the Bianchi constraint (7). In the rest of this paper, we investigate various types of the physical and reference metrics, and identify the ones which are consistent with these conditions.

\section{CONSISTENCY OF BACKGROUND SOLUTIONS WITH DIFFERENT METRIC COMBINATIONS}

\section{A. FLRW metrics with different spatial curvatures: The need for a Lemaitre reference metric}

We begin our investigation of bigravity with nonstandard metric forms by considering the solutions for which both metrics $g_{\mu \nu}$ and $f_{\mu \nu}$ are FLRW with generic spatial curvatures $k_{g}$ and $k_{f}$, where $k_{g}, k_{f}=0$, \pm 1 . The usual background analysis of the cosmology of bigravity assumes $k_{g}=k_{f}$. The reason is partly due to the significant simplification of the calculations in this case, and in addition it seems intuitively reasonable to assume that the two metrics respect the same symmetries and geometries. More complicated cases, however, cannot be excluded a priori. Let us, therefore, leave the choices for $k_{g}$ and $k_{f}$ completely generic and study this case in terms of the consistency of cosmological solutions. Before we continue we note that this case has previously been studied in Ref. [55] and shown to be inconsistent using the Bianchi constraint. In the following, however, we use the implications of the Bianchi constraint in this case as a tool to systematically construct a particular metric combination, FLRW-Lemaitre, which is consistent. Therefore, although our results are in agreement with the findings of Ref. [55], our approach and objectives are different.

With the assumptions made above the most general forms for the metrics are

$$
\begin{aligned}
& g_{\mu \nu} d x^{\mu} d x^{\nu}=-d t^{2}+a^{2}(t) d{\overrightarrow{x_{g}}}^{2}, \\
& f_{\mu \nu} d x^{\mu} d x^{\nu}=-X^{2}(t) d t^{2}+b^{2}(t) d{\overrightarrow{x_{f}}}^{2},
\end{aligned}
$$

where

$$
\begin{aligned}
& d{\overrightarrow{x_{g}}}^{2}=\frac{d r^{2}}{1-k_{g} r^{2}}+r^{2}\left(d \theta^{2}+\sin ^{2}(\theta) d \phi^{2}\right), \\
& d{\overrightarrow{x_{f}}}^{2}=\frac{d r^{2}}{1-k_{f} r^{2}}+r^{2}\left(d \theta^{2}+\sin ^{2}(\theta) d \phi^{2}\right),
\end{aligned}
$$

${ }^{1}$ See, however, Ref. [80], which appeared during the completion of this work, for caveats associated with this rescaling. 
$r, \theta$ and $\phi$ are spherical coordinates, $a$ and $b$ are the scale factors for $g_{\mu \nu}$ and $f_{\mu \nu}$, respectively, and $X$ is the lapse for $f_{\mu \nu}$. Inserting these metric forms into the Bianchi constraint (7) yields the condition

$$
X=\frac{\dot{b}}{\dot{a}} \frac{\beta_{1}(2+\kappa)+2 \beta_{2}(1+2 \kappa) \frac{b}{a}+3 \beta_{3} \kappa\left(\frac{b}{a}\right)^{2}}{3 \beta_{1}+2 \beta_{2}(2+\kappa) \frac{b}{a}+\beta_{3}(1+2 \kappa)\left(\frac{b}{a}\right)^{2}}
$$

on the $f$-metric lapse $X$, where $\kappa \equiv \sqrt{\frac{1-k_{g} r^{2}}{1-k_{f} r^{2}}}$, and an overdot denotes a derivative with respect to $t$. Since the scale factors $a$ and $b$ depend only on time, it is clear from this expression that $X$ cannot be a function of time only and is in general a function of both $t$ and $r$ unless $k_{g}=k_{f}$. This, therefore, shows that FLRW solutions for the two metrics with different spatial curvatures are not allowed, already at the level of the Bianchi constraint.

Now assuming that $X$ is a function of both $t$ and $r$, Eq. (7) places a constraint on the $f$-metric scale factor $b$,

$$
\left(\beta_{3} r X^{\prime}+2(1-\kappa)\left(\beta_{2}+\beta_{3} X\right)\right) b^{2}+2\left(\beta_{2} r X^{\prime}+(1-\kappa)\left(\beta_{1}+\beta_{2} X\right)\right) a b+\beta_{1} r X^{\prime} a^{2}=0,
$$

where a prime denotes a derivative with respect to $r$. We see from this equation that for general choices of the lapse $X$, the scale factor $b$ should also be a function of both $t$ and $r$. It can be shown that the metrics (8) and (9) with both $X$ and $b$ being functions of both $r$ and $t$ cannot be reformulated in FLRW forms by any coordinate transformations (see appendix $\mathrm{A}$ for a detailed proof). We, therefore, conclude that FLRW metrics with different spatial curvatures are not consistent.

Let us now assume that $X$ and $b$ are both functions of $r$ and $t$. Using the Bianchi constraint (7), we arrive at the expressions

$$
\begin{aligned}
X & =\frac{\dot{b}}{\dot{a}} \frac{\beta_{1}(2+\kappa)+2 \beta_{2}(1+2 \kappa) \frac{b}{a}+3 \beta_{3} \kappa\left(\frac{b}{a}\right)^{2}}{3 \beta_{1}+2 \beta_{2}(2+\kappa) \frac{b}{a}+\beta_{3}(1+2 \kappa)\left(\frac{b}{a}\right)^{2}} \\
b^{\prime} & =-\frac{\left(\beta_{3} r X^{\prime}+2(1-\kappa)\left(\beta_{2}+\beta_{3} X\right)\right) b^{2}+2\left(\beta_{2} r X^{\prime}+(1-\kappa)\left(\beta_{1}+\beta_{2} X\right)\right) a b+\beta_{1} r X^{\prime} a^{2}}{2 r\left(\left(\beta_{1}+\beta_{2} X\right) a+\left(\beta_{2}+\beta_{3} X\right) b\right)} .
\end{aligned}
$$

We can, therefore, see that because of different curvatures of reference and physical metrics, the reference metric takes a spherically symmetric and inhomogeneous form, where the lapse and all scale factors are functions of both $r$ and $t$. The most general metric forms corresponding to this case are

$$
\begin{aligned}
& g_{\mu \nu} d x^{\mu} d x^{\nu}=-d t^{2}+a^{2}(t) d{\overrightarrow{x_{g}}}^{2} \\
& f_{\mu \nu} d x^{\mu} d x^{\nu}=-X^{2}(t, r) d t^{2}+Y^{2}(t, r) d r^{2}+Z^{2}(t, r) r^{2} d \Omega^{2},
\end{aligned}
$$

where $d \Omega^{2}=d \theta^{2}+\sin ^{2}(\theta) d \phi^{2}$. The metric $f_{\mu \nu}$ in Eq. (17) has a generic spherically symmetric and inhomogeneous form. In the literature [81, 82], this type of metric is called Lemaitre metric [83], and the cosmological model built on this metric is called Lemaître model. In GR, the Lemaître metric arises when we have inhomogeneous matter sources [82, 84], in particular when the pressure and density are functions of both temporal and spatial coordinates. In the special case of dust or homogenous pressure the Lemaître metric reduces to the so-called Lemaître-Tolman-Bondi (LTB) metric, where the lapse does not depend on the spatial coordinates and can be rescaled.

All our arguments so far for the metrics to take the forms (16) and (17) when we assume unequal curvatures were based only on the Bianchi constraint (7). We can, however, arrive at the same conclusions by analyzing the Einstein equations. Assuming $k_{g} \neq k_{f}$ for the metrics with FLRW forms, we have a nonvanishing $\sqrt{\left(1-k_{g} r^{2}\right) /\left(1-k_{f} r^{2}\right)}$ factor in the Einstein equations for the terms corresponding to the interactions between the two metrics. The interaction part in the $f$-metric Einstein equation plays the role of an inhomogeneous source for $f_{\mu \nu}$, which forces it to take a Lemaître form. For the $g_{\mu \nu}$ metric, there is a coupling to the matter source, and by taking an inhomogeneous matter source one can in principle cancel the inhomogeneities coming from the interaction terms; as a result, $g_{\mu \nu}$ can maintain its homogenous FLRW form. This confirms our finding that the metrics should have the forms (16) and (17) where the matter source is inhomogeneous.

Let us now derive the explicit forms of the Einstein equations for the metrics (16) and (17). As argued above, we assume that the stress-energy-momentum tensor of the matter source coupled to the physical metric has an 
inhomogeneous perfect-fluid form,

$$
\begin{aligned}
& T_{g 0}^{0}=-\rho(t, r), \\
& T_{g 1}^{1}=p(t, r), \\
& T_{g 2}^{2}=p(t, r), \\
& T_{g 3}^{3}=p(t, r),
\end{aligned}
$$

where $\rho$ and $p$ are, respectively, the energy density and pressure for the matter source. For simplicity, here we consider only the $k_{g}=0$ case. The $g$-metric Einstein equations for this case read

$$
\begin{gathered}
3 \frac{\dot{a}^{2}}{a^{2}}+\rho=\beta_{0}+\beta_{1} \frac{(Y+2 Z)}{a}+\beta_{2} \frac{(2 Y+Z) Z}{a^{2}}+\beta_{3} \frac{Y Z^{2}}{a^{3}}, \\
\frac{\dot{a}^{2}}{a^{2}}+2 \frac{\ddot{a}}{a}+p=\beta_{0}+\beta_{1}\left(X+2 \frac{Z}{a}\right)+\beta_{2}\left(2 \frac{X Z}{a}+\frac{Z^{2}}{a^{2}}\right)+\beta_{3} \frac{X Z^{2}}{a^{2}}, \\
\frac{\dot{a}^{2}}{a^{2}}+2 \frac{\ddot{a}}{a}+p=\beta_{0}+\beta_{1}\left(X+\frac{Y+Z}{a}\right)+\beta_{2}\left(\frac{X(Y+Z)}{a}+\frac{Y Z}{a^{2}}\right)+\beta_{3} \frac{X Y Z}{a^{2}} .
\end{gathered}
$$

The equations of motion for $f_{\mu \nu}$ are too unwieldy to be displayed here. One can find the equations for the most general case in Ref. [53]. We see from Eqs. (19)-(21) that with an appropriate choice of $\rho$ and $p$ it is in principle possible to find a function $a(t)$ which satisfies the equations of motion.

In conclusion, our bigravity theory does not allow two FLRW metrics with different spatial curvatures, while it is possible to have a combination of FLRW and Lemaître forms for the metrics $g_{\mu \nu}$ and $f_{\mu \nu}$, respectively, if the matter source takes an inhomogeneous form. In the opposite case of a Lemaitre form for $g_{\mu \nu}$ and an FLRW form for $f_{\mu \nu}$, the equation of motion for $f_{\mu \nu}$ will contain a homogenous Einstein tensor part and an inhomogeneous interaction part. Since $f_{\mu \nu}$ does not couple to matter, the inhomogeneities cannot be cancelled and, therefore, this metric combination in general does not have consistent solutions.

\section{B. FLRW-LTB and LTB-FLRW combinations}

The next possibility we wish to explore is the case where one of the metrics is FLRW and the other one is LTB. For the case where $g_{\mu \nu}$ is FLRW and $f_{\mu \nu}$ is LTB (we denote this as FLRW-LTB), the line elements have the forms

$$
\begin{aligned}
& g_{\mu \nu} d x^{\mu} d x^{\nu}=-d t^{2}+a^{2}(t) d{\overrightarrow{x_{g}}}^{2}, \\
& f_{\mu \nu} d x^{\mu} d x^{\nu}=-X^{2}(t) d t^{2}+Y^{2}(t, r) d r^{2}+Z^{2}(t, r) r^{2} d \Omega^{2},
\end{aligned}
$$

where again $d \Omega^{2}=d \theta^{2}+\sin ^{2}(\theta) d \phi^{2}$. Here we assume that $g_{\mu \nu}$ has a curvature $k_{g}$. In addition we follow the standard recipe for LTB metrics and assume that the physical metric is coupled to a homogeneous perfect-fluid source. In this case the $(0,0)$ and $(1,1)$ components of the $g_{\mu \nu}$ equation of motion become

$$
\begin{gathered}
-3 a \dot{a}^{2}+a^{3}\left(\beta_{0}+\rho\right)+a^{2} \beta_{1}\left(2 Z+Y \sqrt{1-r^{2} k_{g}}\right)+a\left(-3 k_{g}+\beta_{2} Z^{2}+2 \beta_{2} Y Z \sqrt{1-k_{g} r^{2}}\right)+\beta_{3} Y Z^{2} \sqrt{1-k_{g} r^{2}}=0 \\
\dot{a}^{2}+a^{2}\left(p-\beta_{0}-\beta_{1} X\right)-2 a\left(\left(\beta_{1}+\beta_{2} X\right) Z-\ddot{a}\right)+k_{g}-\beta_{2} Z^{2}-\beta_{3} X Z^{2}=0
\end{gathered}
$$

Here we have assumed the stress-energy-momentum tensor for the isotropic and homogeneous perfect fluid to be of the standard form

$$
T_{g \nu}^{\mu}=(\rho+p) u_{0}^{\mu} u_{0 \nu}+p \delta_{\nu}^{\mu}
$$

where $\rho=\rho(t)$ is the rest energy density of the fluid, $p=p(t)$ is its pressure, and $u_{0}^{\mu}$ is its isotropic four-velocity. It is clear from Eq. (25) that in general $Z$ cannot be a function of $r$ since all the other quantities in the equation, including the $f$-metric lapse $X$, are functions only of $t$. If $Z$ is a function of $t$ only, in order to satisfy Eq. (24) $Y$ should be of the form $Y(t, r)=A(t) / \sqrt{1-k_{g} r^{2}}$, where $A(t)$ is an arbitrary function of $t$. This then implies that the reference metric should also be of an FLRW type with the same curvature $k_{g}$. 
In the opposite case, where the physical metric is LTB and the reference metric is FRLW (we denote this as LTB-FLRW), we have

$$
\begin{aligned}
& g_{\mu \nu} d x^{\mu} d x^{\nu}=-d t^{2}+Y^{2}(t, r) d r^{2}+Z^{2}(t, r) r^{2} d \Omega^{2}, \\
& f_{\mu \nu} d x^{\mu} d x^{\nu}=-X^{2}(t) d t^{2}+b^{2}(t) d{\overrightarrow{x_{f}}}^{2} .
\end{aligned}
$$

The $(0,0)$ and $(1,1)$ components of the $f_{\mu \nu}$ equation of motion then read

$$
\begin{gathered}
X^{2} Y\left(\beta_{1} Z^{2}+2 \beta_{2} Z b+\beta_{3} b^{2}\right) \sqrt{1-k_{f} r^{2}}+b\left(\beta_{2} X^{2} Z^{2}+2 \beta_{3} X^{2} Z b+\beta_{4} X^{2} b^{2}-3 k_{f} X^{2}-3 \dot{b}^{2}\right)=0 \\
\beta_{4} b^{2} X^{3}-k_{f} X^{3}+\left(\beta_{1}+\beta_{2} X\right) X^{2} Z^{2}+\beta_{3} X^{2} b^{2}-X \dot{b}^{2}+2 \dot{X} b \dot{b}+2 b X^{2}\left(\beta_{2}+\beta_{3} X\right) Z-2 X b \ddot{b}=0 .
\end{gathered}
$$

Similarly to the case for $g_{\mu \nu}$, here we again see from Eq. (30) that $Z$ in general cannot depend on $r$. Equation (29), therefore, implies that $Y$ should have the form $Y(t, r)=B(t) / \sqrt{1-k_{f} r^{2}}$, where $B(t)$ is again an arbitrary function of $t$. This form for $Y$ then brings the physical metric into an FLRW type, again with the same curvature as the reference metric.

It is important to note that in both metric combinations discussed above the Bianchi constraint is not satisfied, and since we get the Bianchi constraint using the covariant conservation of the stress-energy-momentum tensor, we can therefore state that in general neither FLRW-LTB nor LTB-FLRW can occur for any choices of covariantly conserved $T_{\mu \nu}^{g}$.

\section{LTB-LTB combination}

Let us now study the case where both metrics $g_{\mu \nu}$ and $f_{\mu \nu}$ are of LTB forms (we denote this as LTB-LTB),

$$
\begin{aligned}
& g_{\mu \nu} d x^{\mu} d x^{\nu}=-d t^{2}+A^{2}(t, r) d r^{2}+B^{2}(t, r) d \Omega^{2}, \\
& f_{\mu \nu} d x^{\mu} d x^{\nu}=-X^{2}(t) d t^{2}+Y^{2}(t, r) d r^{2}+Z^{2}(t, r) d \Omega^{2} .
\end{aligned}
$$

In this case the 0 component of the Bianchi constraint (7) enforces the $f$-metric lapse $X(t)$ to satisfy the equation

$$
X=\frac{2 U \dot{Z}+V \dot{Y}}{2 U \dot{B}+V \dot{A}}
$$

where

$$
\begin{aligned}
& U(t, r) \equiv B(t, r)\left(\beta_{1} A(t, r)+\beta_{2} Y(t, r)\right)+Z(t, r)\left(\beta_{2} A(t, r)+\beta_{3} Y(t, r)\right), \\
& V(t, r) \equiv \beta_{1} B^{2}(t, r)+2 \beta_{2} B(t, r) Z(t, r)+\beta_{3} Z^{2}(t, r) .
\end{aligned}
$$

The constraint (33) holds when

$$
\begin{aligned}
B(t, r) & =\widetilde{B}(t) s(r), \\
Z(t, r) & =\widetilde{Z}(t) s(r), \\
A(t, r) & =\widetilde{A}(t) q(r), \\
Y(t, r) & =\widetilde{Y}(t) q(r),
\end{aligned}
$$

where $s$ and $q$ are functions of $r$ only, obtained through solving Einstein equations. In addition, the (1,2) component of the equation of motion for $g_{\mu \nu}$ becomes

$$
B^{\prime} \dot{A}=A \dot{B}^{\prime}
$$

For $f_{\mu \nu}$ the corresponding equation is

$$
Z^{\prime} \dot{Y}=Y \dot{Z}^{\prime}
$$

From these equations we obtain the following relations between $\widetilde{B}(t), \widetilde{A}(t), \widetilde{Z}(t)$, and $\widetilde{Y}(t)$ :

$$
\begin{aligned}
& \widetilde{B}(t) \dot{\widetilde{A}}(t)=\widetilde{A}(t) \dot{\widetilde{B}}(t), \\
& \widetilde{Z}(t) \dot{\widetilde{Y}}(t)=\widetilde{Y}(t) \dot{\widetilde{Z}}(t) .
\end{aligned}
$$


We, therefore, find that $\widetilde{A}(t)=C_{1} \widetilde{B}(t)$ and $\widetilde{Y}(t)=C_{2} \widetilde{Z}(t)$, where $C_{1}$ and $C_{2}$ are some arbitrary constants. We obtain another useful constraint on our functions from the $(2,2)$ component of the $g_{\mu \nu}$ equation of motion,

$$
-2 B\left(\left(\beta_{1}+\beta_{2} X\right) Z-\ddot{B}\right)+\dot{B}^{2}-B^{2}\left(\beta_{0}+\beta_{1} X-p\right)-\left(\beta_{2}+\beta_{3} X\right) Z^{2}=\frac{B^{\prime 2}}{A^{2}}-1 .
$$

It is easy to see that the $r$-dependent part of the left-hand side of Eq. (44) is $s^{2}(r)$. The right-hand side of Eq. (44) should also have the same dependence on $r$ in order for the $r$ dependence of both sides of the equation to cancel out. Therefore, in this case we have

$$
\frac{B^{\prime 2}}{A^{2}}-1=\frac{s^{\prime 2}}{q^{2}}-1=C_{3} s^{2},
$$

where $C_{3}$ is another arbitrary constant. From the $(3,3)$ component of the equation of motion for $g_{\mu \nu}$ we find

$$
s^{\prime} q^{\prime}-q s^{\prime \prime}=C_{4} s q^{3} .
$$

Equations (45) and (46) are the conditions which should be fulfilled by $s$ and $q$ for the consistency of the Einstein equations for both $g_{\mu \nu}$ and $f_{\mu \nu}$. In summary, we find that the LTB-LTB combination is consistent only for particular subclasses of LTB metrics which satisfy all the conditions stated above.

\section{Bianchi I-FLRW combination}

We would also like to investigate the cases where one of the two metrics is homogeneous but anisotropic while the other one is both homogeneous and isotropic (i.e. has an FLRW form). Here we consider only Bianchi type I models, which are the simplest anisotropic models and capture most of the interesting anisotropic effects. The general properties of the cases where both metrics are anisotropic, simultaneously diagonal, and of the same Bianchi types within the Bianchi class A, which includes types I, II, $\mathrm{VI}_{0}, \mathrm{VII}_{0}$, VIII, and IX, are discussed in Ref. 79]; we, therefore, do not consider those cases in this paper. As discussed in Sec. I. our main motivation for studying nonFLRW solutions in bigravity is the potential resolution of the problems with the standard scenario, in particular the instability issues, in this framework. However, there are also theoretical and observational arguments [85] in support of an anisotropic phase in the early Universe which approached isotropy at later times. It is, therefore, interesting also from this perspective to see whether such solutions are allowed in bigravity (see Ref. [79] for other motivations for studying anisotropies in bigravity, including a potentially interesting connection to dark matter).

Bianchi type I, or simply Bianchi I, models are spatially homogenous and flat but the expansion rate is directiondependent. In GR, these models have been studied for different sources with the equation of state $p=\omega \rho$. It has been shown in Ref. [86] that for cases with $\omega<1$ the anisotropic models evolve towards an FLRW universe, while for $\omega=1$ the process of isotropization does not take place. In the present study of Bianchi metrics we do not consider the question of isotropization and only investigate the consistency of such solutions in terms of the field equations and Bianchi constraint for both isotropic and anisotropic perfect-fluid sources.

In this section we focus on the case where the physical metric is assumed to be of an anisotropic Bianchi I form while the reference metric is FLRW and flat; we call this case Bianchi I-FLRW. We show that for any choices of the matter source, isotropic or anisotropic, the Bianchi I-FLRW combination does not satisfy the conditions of the theory and is, therefore, not a consistent solution.

The metrics for this particular case have the forms

$$
\begin{aligned}
& g_{\mu \nu} d x^{\mu} d x^{\nu}=-d t^{2}+a_{1}^{2}(t) d x^{2}+a_{2}^{2}(t) d y^{2}+a_{3}^{2}(t) d z^{2}, \\
& f_{\mu \nu} d x^{\mu} d x^{\nu}=-X^{2}(t) d t^{2}+b^{2}(t) d \vec{x}^{2},
\end{aligned}
$$

where $d \vec{x}^{2}=d x^{2}+d y^{2}+d z^{2}$, and $x, y$, and $z$ are Cartesian coordinates. Here, $a_{1}, a_{2}$, and $a_{3}$ are the $g$-metric scale factors along different directions, and $b$ is the $f$-metric scale factor. The equation of motion for $f_{\mu \nu}$ gives, independently of the matter source for the $g$ metric,

$$
\begin{array}{r}
X^{2}\left(\beta_{1} a_{1} a_{2} a_{3}+\beta_{2}\left(a_{1} a_{3}+a_{1} a_{2}+a_{2} a_{3}\right) b+\beta_{3}\left(a_{1}+a_{2}+a_{3}\right) b^{2}+\beta_{4} b^{3}\right)-3 b \dot{b}^{2}=0, \\
X^{2}\left(a_{3}\left(a_{2}\left(\beta_{1}+\beta_{2} X\right)+b\left(\beta_{2}+\beta_{3} X\right)\right)+b\left(a_{2}\left(\beta_{2}+\beta_{3} X\right)+b\left(\beta_{3}+\beta_{4} X\right)\right)\right)-X\left(2 b \ddot{b}+\dot{b}^{2}\right)+2 b \dot{X} \dot{b}=0, \\
X^{2}\left(a_{3}\left(a_{1}\left(\beta_{1}+\beta_{2} X\right)+b\left(\beta_{2}+\beta_{3} X\right)\right)+b\left(a_{1}\left(\beta_{2}+\beta_{3} X\right)+b\left(\beta_{3}+\beta_{4} X\right)\right)\right)-X\left(2 b \ddot{b}+\dot{b}^{2}\right)+2 b \dot{X} \dot{b}=0, \\
X^{2}\left(a_{2}\left(a_{1}\left(\beta_{1}+\beta_{2} X\right)+b\left(\beta_{2}+\beta_{3} X\right)\right)+b\left(a_{1}\left(\beta_{2}+\beta_{3} X\right)+b\left(\beta_{3}+\beta_{4} X\right)\right)\right)-X\left(2 b \ddot{b}+\dot{b}^{2}\right)+2 b \dot{X} \dot{b}=0 .
\end{array}
$$


From Eqs. (501) and (51) we see that $a_{1}=a_{2}$, while Eqs. (51) and (52) imply that $a_{2}=a_{3}$; therefore $a_{1}=a_{2}=a_{3}$. This means that the physical metric is forced to become isotropic. Therefore, using only the $f$-metric equation of motion and no other equations or conditions of the theory, we can conclude that one cannot have a Bianchi I physical metric while the reference metric maintains its FLRW form. Since $f_{\mu \nu}$ is not sourced by matter, our conclusion is general and independent of what form the matter source takes.

\section{E. FLRW-Bianchi I combination}

\section{Isotropic source}

Let us now assume that the reference metric takes the Bianchi-I anisotropic form while the physical metric is FLRW and flat; we call this case FLRW-Bianchi I. This means that each direction has a different scale factor in the $f$ metric. The metrics, therefore, possess the forms

$$
\begin{aligned}
& g_{\mu \nu} d x^{\mu} d x^{\nu}=-d t^{2}+a^{2}(t) d \vec{x}^{2}, \\
& f_{\mu \nu} d x^{\mu} d x^{\nu}=-X^{2}(t) d t^{2}+b_{1}^{2}(t) d x^{2}+b_{2}^{2}(t) d y^{2}+b_{3}^{2}(t) d z^{2},
\end{aligned}
$$

where again $d \vec{x}^{2}=d x^{2}+d y^{2}+d z^{2}$. Here, $a$ is the scale factor for $g_{\mu \nu}$, and $b_{1}, b_{2}$ and $b_{3}$ are the $f$-metric scale factors along different directions. In addition, let us assume that the matter source, which couples to the physical metric $g_{\mu \nu}$, is a homogeneous and isotropic perfect fluid. The stress-energy-momentum tensor has, therefore, the form given in Eq. (26).

For the metric forms (53) and (54), the $g$-metric Einstein equation (3) reads

$$
\begin{gathered}
3 \frac{\dot{a}^{2}}{a^{2}}-\rho=\beta_{0}+\beta_{1} \frac{b_{1}+b_{2}+b_{3}}{a}+\beta_{2} \frac{b_{1} b_{2}+b_{1} b_{3}+b_{2} b_{3}}{a^{2}}+\beta_{3} \frac{b_{1} b_{2} b_{3}}{a^{3}}, \\
\frac{\dot{a}^{2}}{a^{2}}+2 \frac{\ddot{a}}{a}+p=\beta_{0}+\beta_{1}\left(X+\frac{b_{2}+b_{3}}{a}\right)+\beta_{2}\left(\frac{X\left(b_{2}+b_{3}\right)}{a}+\frac{b_{2} b_{3}}{a^{2}}\right)+\beta_{3} \frac{X b_{2} b_{3}}{a^{2}}, \\
\frac{\dot{a}^{2}}{a^{2}}+2 \frac{\ddot{a}}{a}+p=\beta_{0}+\beta_{1}\left(X+\frac{b_{1}+b_{3}}{a}\right)+\beta_{2}\left(\frac{X\left(b_{1}+b_{3}\right)}{a}+\frac{b_{1} b_{3}}{a^{2}}\right)+\beta_{3} \frac{X b_{1} b_{3}}{a^{2}}, \\
\frac{\dot{a}^{2}}{a^{2}}+2 \frac{\ddot{a}}{a}+p=\beta_{0}+\beta_{1}\left(X+\frac{b_{1}+b_{2}}{a}\right)+\beta_{2}\left(\frac{X\left(b_{1}+b_{2}\right)}{a}+\frac{b_{1} b_{2}}{a^{2}}\right)+\beta_{3} \frac{X b_{1} b_{2}}{a^{2}} .
\end{gathered}
$$

It is straightforward to see from these equations that $b_{1}, b_{2}$, and $b_{3}$ are equal. This means that if we initially assume an anisotropic form for the reference metric while the physical metric and the matter source are both assumed to be isotropic, the structure of the Einstein equations automatically forces the reference metric to also be isotropic.

\section{Anisotropic source}

Let us now relax the isotropy condition on the matter source and study the FLRW-Bianchi I scenario when the physical metric is coupled to a source which is anisotropic and of a Bianchi I type. The stress-energy-momentum tensor in this case takes the form

$$
\begin{aligned}
& T_{g 0}^{0}=-\rho(t), \\
& T_{g 1}^{1}=p_{1}(t), \\
& T_{g 2}^{2}=p_{2}(t), \\
& T_{g 3}^{3}=p_{3}(t),
\end{aligned}
$$

where the components of the fluid pressure, $p_{1}, p_{2}$, and $p_{2}$, are allowed to be different along different directions and therefore create anisotropy in the fluid.

It follows from the Bianchi constraint (7) that in this case the $f$-metric lapse $X(t)$ is given by (here all the variables depend only on $t$ )

$$
X=\frac{1}{\dot{a}} \frac{\beta_{1} a^{2}\left(b_{1}+b_{2}+b_{3}\right)^{\cdot}+\beta_{2} a\left(b_{1} b_{2}+b_{1} b_{3}+b_{2} b_{3}\right)^{\cdot}+\beta_{3}\left(b_{1} b_{2} b_{3}\right)^{\cdot}}{3 \beta_{1} a^{2}+2 \beta_{2} a\left(b_{1}+b_{2}+b_{3}\right)+\beta_{3}\left(b_{1} b_{2}+b_{1} b_{3}+b_{2} b_{3}\right)}
$$


The Einstein equation for $g_{\mu \nu}$ gives

$$
\begin{aligned}
3 \frac{\dot{a}^{2}}{a^{2}}-\rho & =\beta_{0}+\beta_{1} \frac{b_{1}+b_{2}+b_{3}}{a}+\beta_{2} \frac{b_{1} b_{3}+b_{1} b_{2}+b_{2} b_{3}}{a^{2}}+\beta_{3} \frac{b_{1} b_{2} b_{3}}{a^{3}}, \\
\frac{\dot{a}^{2}}{a^{2}}+2 \frac{\ddot{a}}{a}+p_{1} & =\beta_{0}+\beta_{1} X+\left(\beta_{2}+\beta_{3} X\right) \frac{b_{2} b_{3}}{a^{2}}+\left(\beta_{1}+\beta_{2} X\right) \frac{b_{2}+b_{3}}{a}, \\
\frac{\dot{a}^{2}}{a^{2}}+2 \frac{\ddot{a}}{a}+p_{2} & =\beta_{0}+\beta_{1} X+\left(\beta_{2}+\beta_{3} X\right) \frac{b_{1} b_{3}}{a^{2}}+\left(\beta_{1}+\beta_{2} X\right) \frac{b_{3}+b_{1}}{a}, \\
\frac{\dot{a}^{2}}{a^{2}}+2 \frac{\ddot{a}}{a}+p_{3} & =\beta_{0}+\beta_{1} X+\left(\beta_{2}+\beta_{3} X\right) \frac{b_{1} b_{2}}{a^{2}}+\left(\beta_{1}+\beta_{2} X\right) \frac{b_{2}+b_{1}}{a},
\end{aligned}
$$

and the $f$-metric Einstein equation gives

$$
\begin{aligned}
& \beta_{1} a^{3} X^{2}+\beta_{2} a^{2} X^{2}\left(b_{1}+b_{2}+b_{3}\right)+\beta_{3} a X^{2}\left(b_{1} b_{2}+b_{1} b_{3}+b_{2} b_{3}\right)+\beta_{4} b_{1} b_{2} b_{3} X^{2}-b_{3} \dot{b_{1}} \dot{b_{2}}-b_{1} \dot{b_{2}} \dot{b_{3}}-b_{2} \dot{b_{1}} \dot{b_{3}}=0, \\
& a^{2} X^{2}\left(\beta_{1}+\beta_{2} X\right)+a X^{2}\left(\beta_{2}+\beta_{3} X\right)\left(b_{2}+b_{3}\right)+b_{3}\left(\dot{X} \dot{b_{2}}+X^{2}\left(\beta_{3}+\beta_{4} X\right) b_{2}\right)+b_{2} \dot{X} \dot{b_{3}}-X\left(\dot{b_{2}} \dot{b_{3}}+\ddot{b_{3}} \ddot{b_{2}}+b_{2} \ddot{b_{3}}\right)=0 \text {, } \\
& a^{2} X^{2}\left(\beta_{1}+\beta_{2} X\right)+a X^{2}\left(\beta_{2}+\beta_{3} X\right)\left(b_{1}+b_{3}\right)+b_{3}\left(\dot{X} \dot{b_{1}}+X^{2}\left(\beta_{3}+\beta_{4} X\right) b_{1}\right)+b_{1} \dot{X} \dot{b_{3}}-X\left(\dot{b_{1}} \dot{b_{3}}+b_{3} \ddot{b_{1}}+b_{1} \ddot{b_{3}}\right)=0 \text {, } \\
& a^{2} X^{2}\left(\beta_{1}+\beta_{2} X\right)+a X^{2}\left(\beta_{2}+\beta_{3} X\right)\left(b_{1}+b_{2}\right)+b_{2}\left(\dot{X} \dot{b_{1}}+X^{2}\left(\beta_{3}+\beta_{4} X\right) b_{1}\right)+b_{1} \dot{X} \dot{b_{2}}-X\left(\dot{b_{1}} \dot{b_{2}}+\ddot{b_{2}} \ddot{b_{1}}+b_{1} \ddot{b_{2}}\right)=0 \text {. }
\end{aligned}
$$

From Eqs. (64)- (67) we see that because of the anisotropic matter source, $b_{1}, b_{2}$, and $b_{3}$ satisfy different algebraic equations and can, therefore, be different from each other. Equations (68) form a set of second-order differential equations with respect to $b_{1}, b_{2}$, and $b_{3}$. Each equation has two independent solutions and, therefore, in spite of the fact that $b_{1}, b_{2}$, and $b_{3}$ satisfy the same differential equation, in general they can be different. We can, therefore, conclude that the combination FRLW-Bianchi I in the presence of an anisotropic matter source is in principle possible.

\section{CONSISTENCY OF SOLUTIONS WITH ONLY ONE PERTURBED METRIC}

In the previous sections we explored various combinations of cosmologically interesting metric types for the two metrics of the theory of massive bigravity. Our investigation was, however, only at the level of the background dynamics of the Universe. In this section we study some other interesting cases where perturbations around the background metrics are considered.

Perturbations are clearly crucial for cosmology and it is, therefore, interesting to ask whether there are any consistent, nonstandard ways to perturb the metrics. In all the previous works on the perturbative analysis of bigravity a standard recipe has been followed: the background metrics have been assumed to be FLRW and both the physical and reference metrics have then been perturbed around the FLRW solutions. As stated in Sec. [ our main motivation in this paper has been to try alternative solutions which might be free of various instabilities which appear in bigravity models at the level of perturbations. It would, therefore, be interesting if one could avoid the instabilities by finding alternative ways of perturbing the metrics. In addition, the usual perturbation equations are very complicated and it would be very helpful if one could find a way to simplify the equations. One of such approaches could be to work with solutions which leave the reference metric unperturbed while the physical metric is perturbed as usual. This scenario is physically justified because the reference metric does not couple to the matter components and cannot be measured directly from observations; the reference metric affects our observables only through its interactions with the physical metric. In what follows we analyze the consistency of this possibility using both scalar and tensor perturbations.

Let us start with scalar perturbations up to linear order for both metrics. For simplicity we assume both metrics to be of the flat FLRW type. Including only scalar perturbations and using the notations of Ref. [68], the line elements for the perturbed physical and reference metrics $g_{\mu \nu}$ and $f_{\mu \nu}$ have the forms

$$
\begin{aligned}
& d s_{g}^{2}=a^{2}(\eta)\left[-\left(1+2 \Psi_{g}\right) d \eta^{2}+2 \partial_{i} B_{g} d x^{i} d \eta+\left[\left(1-2 \Phi_{g}\right) \delta_{i j}+2 \partial_{i} \partial_{j} E_{g}\right] d x^{i} d x^{j}\right], \\
& d s_{f}^{2}=b^{2}(\eta)\left[-\left(1+2 \Psi_{f}\right) X^{2} d \eta^{2}+2 \partial_{i} B_{f} X d x^{i} d \eta+\left[\left(1-2 \Phi_{f}\right) \delta_{i j}+2 \partial_{i} \partial_{j} E_{f}\right] d x^{i} d x^{j}\right] .
\end{aligned}
$$

Here $a$ and $b$ are the scale factors corresponding to $g_{\mu \nu}$ and $f_{\mu \nu}$, respectively, $X$ is the lapse for $f_{\mu \nu}$, and the perturbation quantities $\left\{\Psi_{g, f}, B_{g, f}, \Phi_{g, f}, E_{g, f}\right\}$ are allowed to depend on both conformal time $\eta$ and space. Spatial indices are raised and lowered by the Kronecker delta. In this section a dot denotes a derivative with respect to the conformal time $\eta$. 
In order to write down the expression for the perturbed matter stress-energy-momentum tensor, we assume a perfect fluid with an equation of state $p=w \rho$. In addition, we describe the matter perturbations with only one scalar field $\chi$. This procedure has been proposed in Ref. [87]. With these assumptions and conventions we have

$$
\begin{aligned}
\delta T^{0}{ }_{0} & =-(\rho+p)\left(3 \Phi_{g}-E_{g, l l}-\chi_{, l l}\right), \\
\delta T^{i}{ }_{0} & =-(\rho+p) \dot{\chi}^{, i}, \\
\delta T^{0}{ }_{i} & =(\rho+p)\left(B_{g, i}+\dot{\chi}_{, i}\right), \\
\delta T^{i}{ }_{j} & =w(\rho+p)\left(3 \Phi_{g}-E_{g, l l}-\chi_{, l l}\right) \delta^{i}{ }_{j},
\end{aligned}
$$

where we sum over indices $l$. Following Ref. [69] and after some useful gauge fixing and transformations developed in Ref. [88], we set $\Phi_{f}=\chi=0$ and arrive at the first-order perturbation equations

$$
\begin{aligned}
& 2 \mathcal{H}\left(3 \dot{\Phi}_{g}+k^{2} \dot{E}_{g}\right)+a^{2}\left((1+w) \rho\left(3 \Phi_{g}+k^{2} E_{g}\right)+r Z\left(3 \Phi_{g}+k^{2}\left(E_{g}-E_{f}\right)\right)\right) \\
& +2\left(k^{2} \Phi_{g}+\mathcal{H}\left(3 \mathcal{H} \Psi_{g}-k^{2} B_{g}\right)\right)=0, \\
& 2(X+1) \dot{\Phi}_{g}+2 \mathcal{H}(X+1) \Psi_{g}-Z r\left(X B_{f}-B_{g}\right)+(1+w) \rho(1+X) B_{g}=0, \\
& 2\left(k^{2} \ddot{E}_{g}+3 \ddot{\Phi}_{g}\right)+2 \mathcal{H}\left(3 \dot{\Psi}_{g}+6 \dot{\Phi}_{g}+2 k^{2} \dot{E}_{g}\right)-2 k^{2} \dot{B}_{g}+3 Z a^{2} r X\left(\Psi_{f}+\Psi_{g}\right) \\
& +a^{2}\left(-3(1+w) \rho\left(2 \Psi_{g}+w\left(3 \Phi_{g}+k^{2} E_{g}\right)\right)+2 r\left(-3 Z \Psi_{g}+\tilde{Z}\left(3 \Phi_{g}+k^{2}\left(E_{g}-E_{f}\right)\right)\right)\right) \\
& +2\left(9 \mathcal{H}^{2}-k^{2}\right) \Psi_{g}+2 k^{2}\left(\Phi_{g}-2 \mathcal{H} B_{g}\right)=0, \\
& \ddot{E}_{g}-\dot{B}_{g}+2 \mathcal{H} \dot{E}_{g}+\tilde{Z} a^{2} r\left(E_{g}-E_{f}\right)-\Psi_{g}-2 \mathcal{H} B_{g}+\Phi_{g}=0, \\
& 2 r \mathcal{H}_{f} k^{2} \dot{E}_{f}-a^{2} Z X^{2}\left(k^{2} E_{g}-k^{2} E_{f}+3 \Phi_{g}\right)-2 r X \mathcal{H}_{f} k^{2} B_{f}+6 \mathcal{H}_{f}^{2} r \Psi_{f}=0, \\
& 2 \mathcal{H}_{f} r(X+1) \Psi_{f}+X a^{2} Z\left(X B_{f}-B_{g}\right)=0, \\
& r X \ddot{E}_{f}-r\left(-2 X \mathcal{H}_{f}+\dot{X}_{)} \dot{E}_{f}-X^{2}\left(\dot{B}_{f} r+r X \Psi_{f}+2 r \mathcal{H}_{f} B_{f}+a^{2} \tilde{Z}\left(E_{g}-E_{f}\right)\right)=0,\right.
\end{aligned}
$$

where we have defined

$$
\begin{aligned}
Z & \equiv \beta_{1}+2 \beta_{2} r+\beta_{3} r^{2} \\
\tilde{Z} & \equiv \beta_{1}+\beta_{2} r(1+X)+\beta_{3} r^{2} X, \\
r & \equiv \frac{b}{a}, \quad \mathcal{H} \equiv \frac{\dot{a}}{a} \quad \mathcal{H}_{f} \equiv \frac{\dot{b}}{b} .
\end{aligned}
$$

Note that $r$ in this section is not the radial coordinate. Let us now assume that only the physical metric $g_{\mu \nu}$ is perturbed; i.e., all the perturbative quantities for $f_{\mu \nu},\left\{\Psi_{f}, B_{f}, \Phi_{f}, E_{f}\right\}$, are vanishing. Looking at Eqs. (76)-(78) we find

$$
\begin{aligned}
X^{2} a^{2} Z\left(k^{2} E_{g}+3 \Phi_{g}\right) & =0, \\
X a^{2} Z B_{g} & =0, \\
X^{2} a^{2} \tilde{Z} E_{g} & =0 .
\end{aligned}
$$

From Eqs. (82)-(84) it is clear that the $g$-metric scalar perturbations, $B_{g}, \Phi_{g}$ and $E_{g}$, should vanish (which in turn implies, using Eq. (75), that $\Psi_{g}$ should also be vanishing) unless the quantities $\tilde{Z}$ and $Z$ are both vanishing. In order to prove that the latter cannot be the case let us now assume $\tilde{Z}=0$. In this case we have

$$
\beta_{1}+\beta_{2} r(1+X)+\beta_{3} r^{2} X=0 .
$$

On the other hand, we know from the Bianchi constraint (7) that at the background level $X$ satisfies the condition

$$
X=\frac{a}{\dot{a}} \frac{\dot{b}}{b}
$$

If we now insert the value of $X$ from Eq. (86) into Eq. (85) we obtain

$$
\beta_{1} a \dot{a}+\beta_{2}(a \dot{b}+b \dot{a})+\beta_{3} b \dot{b}=0 \Longrightarrow \frac{\beta_{1}}{2}\left(a^{2}\right)^{\cdot}+\beta_{2}(a b)^{\cdot}+\frac{\beta_{3}}{2}\left(b^{2}\right)^{\cdot}=0 .
$$


Finally, by integrating Eq. (87) over conformal time we get

$$
\beta_{1} a^{2}+2 \beta_{2} a b+\beta_{3} b^{2}=C \quad \Longrightarrow \quad \beta_{1}+2 \beta_{2} r+\beta_{3} r^{2}=\frac{C}{a^{2}},
$$

where $C$ is an arbitrary constant. We, however, need to assume $Z=0$ in this case, which then implies $C=0$. The condition (88) with $C=0$ requires $r$ to be a constant and given by a particular combination of the parameters $\beta_{1}, \beta_{2}$ and $\beta_{3}$. From the background equations of bimetric gravity [68] one realizes immediately that this condition implies that the Universe is in a de Sitter state (or, trivially, that $a=b=0$ ).

Let us now study the opposite scenario, i.e. where only the reference metric $f_{\mu \nu}$ is perturbed and the physical metric remains unperturbed. In this case the $g$-metric perturbations $\left\{\Psi_{g}, B_{g}, \Phi_{g}, E_{g}\right\}$ are vanishing. Now Eqs. (72)-(75) imply

$$
\begin{aligned}
Z r a^{2} k^{2} E_{f} & =0, \\
Z r X B_{f} & =0, \\
3 Z a^{2} r X \Psi_{f}-2 \tilde{Z} a^{2} r k^{2} E_{f} & =0 \\
\tilde{Z} a^{2} r E_{f} & =0 .
\end{aligned}
$$

From Eqs. (89)-(92) we can again conclude that the $f$-metric scalar perturbations should also vanish in this case (note that $\Phi_{f}$ is already vanishing due to our gauge choice), unless both $\tilde{Z}$ and $Z$ are vanishing. The latter case again corresponds to a de Sitter universe where $r=$ const. We can, therefore, conclude, based on both cases studied here, that for any cosmological configurations different from a pure de Sitter universe, the possibility that only one of the two metrics is perturbed is excluded at the scalar level.

A similar analysis can be done using tensor perturbations. The line elements for the physical and reference metrics in this case take the forms

$$
\begin{aligned}
& d s_{g}^{2}=a^{2}(\eta)\left[-d \eta^{2}+\left(\delta_{i j}+h_{i j}^{g}\right) d x^{i} d x^{j}\right], \\
& d s_{f}^{2}=b^{2}(\eta)\left[-X^{2} d \eta^{2}+\left(\delta_{i j}+h_{i j}^{f}\right) d x^{i} d x^{j}\right],
\end{aligned}
$$

where $h_{i j}^{g, f}$ are the tensor perturbation quantities and are in general functions of space and conformal time $\eta$. These quantities satisfy the relations

$$
h_{i}^{i}=0, \quad h_{i j}^{i}=0,
$$

for both $h^{g}$ and $h^{f}$; here we again use the Kronecker delta to raise or lower spatial indices.

In a perfect-fluid model there are no tensor perturbation modes in the stress-energy-momentum tensor, and as a result, the right-hand side of the perturbed Einstein equation for $g_{\mu \nu}$ vanishes. Because of the conditions (95) we have only two degrees of freedom for each $h_{i j}$ which are fully decoupled and satisfy the following first-order perturbation equations in Fourier space:

$$
\begin{aligned}
& \ddot{h^{g}}+2 \mathcal{H} \dot{h^{g}}+k^{2} h^{g}+a^{2} r \tilde{Z}\left(h^{g}-h^{f}\right)=0, \\
& \ddot{h^{f}}+\left(2 \mathcal{H}_{f}-\frac{\dot{X}}{X}\right) \dot{h^{f}}+k^{2} X^{2} h^{f}+\frac{a^{2} X}{r} \tilde{Z}\left(h^{f}-h^{g}\right)=0 .
\end{aligned}
$$

As in the scalar case discussed above, we now assume that only the $g$ metric is perturbed while the $f$ metric is unperturbed (the argument is identical if we assume instead that only $f_{\mu \nu}$ is perturbed); this means that $h^{f}=0$. From Eq. (97) it is straightforward to see that the $g$-metric tensor perturbation $h^{g}$ should also vanish, unless the quantity $\tilde{Z}$ is vanishing. This corresponds to the condition (88), but contrary to the scalar case, here $C$ is an arbitrary constant and is not required to vanish in order for the $g$ and $f$ tensor perturbations to decouple. One obvious solution is, however, again the case where $C=0$, i.e. a purely de Sitter universe which does not correspond to the real universe. The only nontrivial class of solutions for which the tensor modes decouple while the scalar modes are still coupled is the case where $C$ is nonvanishing. Whether or not these solutions are cosmologically interesting remains to be investigated; we leave this for future work 2

2 The case for vector perturbations is not of interest to us here, but looking at the corresponding perturbative equations presented in Ref. 88] it seems to us that the case where vector perturbations are nonvanishing only for the physical metric while the reference metric remains unperturbed is possible. 


\begin{tabular}{|c|c|c|c|c|}
\hline \multicolumn{5}{|c|}{ Metric combinations } \\
\hline$g_{\mu \nu}$ (physical metric) & $f_{\mu \nu}$ (reference metric) & $T_{\mu \nu}^{g}$ & Possibility & Reference \\
\hline \hline FLRW $(k)$ & FLRW $(k)$ & PF & $\checkmark$ & Standard \\
\hline FLRW $\left(k_{g}\right)$ & FLRW $\left(k_{f}\right)$ & PF & $\times$ & Present work and Ref. [55] \\
\hline FLRW & Lemaitre & PF & $\times$ & Present work \\
\hline FLRW & Lemaître & Inhom. & $\checkmark$ & Present work and Ref. [26] \\
\hline Lemaître & FLRW & Any & $\times$ & Present work \\
\hline FLRW & LTB & Any & $\times$ & Present work \\
\hline LTB & FLRW & Any & $\times$ & Present work \\
\hline LTB & LTB & PF & $\checkmark^{\mathrm{a}}$ & Present work \\
\hline Bianchi I & Bianchi I & Any & $\times$ & Present work \\
\hline FLRW & Bianchi I & Aniso. & $\checkmark$ & Present work \\
\hline FLRW & Bianchi Class A & PF & $\checkmark$ & Present work \\
\hline Bianchi Class A & Perturbed FLRW & Perturbed PF & $\checkmark$ & \\
\hline Perturbed FLRW & Standard \\
\hline Perturbed FLRW (scalars) & Unperturbed FLRW (scalars) & Perturbed PF & $\times^{\mathrm{b}}$ & Present work \\
\hline Unperturbed FLRW (scalars) & Perturbed FLRW (scalars) & Perturbed PF & $\times^{\mathrm{b}}$ & Present work \\
\hline Perturbed FLRW (tensors) & Unperturbed FLRW (tensors) & Perturbed PF & $\times^{\mathrm{c}}$ & Present work \\
\hline Unperturbed FLRW (tensors) & Perturbed FLRW (tensors) & Perturbed PF & $\times^{\mathrm{c}}$ & Present work \\
\hline
\end{tabular}

a There are conditions which must be satisfied; see the text for details.

b Unless the metrics are de Sitter; see the text for details.

c Unless the metrics are de Sitter or satisfy a specific condition; see the text for details.

Table I. List of all metric combinations studied in the present work, as well as a few other interesting combinations studied in the literature. $\checkmark$ and $\times$ denote consistent and inconsistent cases, respectively. Here, "PF," "Inhom." and "Aniso." stand, respectively, for isotropic and homogeneous (perfect fluid), isotropic but inhomogeneous, and homogeneous but anisotropic matter sources. "Any" stands for a matter source of any type. $k$ is the spatial curvature of an FLRW metric; in cases with explicit indices $g$ and $f$ for $k$ the two metrics are assumed to have different spatial curvatures.

\section{CONCLUSIONS}

One of the problems of contemporary cosmology is that there are very few, if any, well-studied, and well-motivated alternatives to the standard model that are viable and distinguishable from $\Lambda$ CDM. Massive bigravity models (e.g. the particular model discussed in [68]) could be one of such rare cases if the problem of perturbation instability ([69, 70]) could be overcome. However, most studies of bigravity models confined themselves to homogeneous and isotropic metrics with no or identical spatial curvatures. Before ruling out bigravity cosmology one should, therefore, see if the problems could be solved or alleviated when a different geometry is chosen. This paper was devoted to addressing this question by scanning several relatively simple possible metric combinations in search for consistent cases. Future work will be necessary to actually solve the equations for some of such cases and identify cosmologically viable ones.

We, however, found that there are only a few metric combinations that survive our analysis. In most cases, two different metrics are impossible unless a suitable modification is made to the matter source beyond the standard perfect-fluid assumption. Our results are summarized in Table I. In particular we find that the only alternative combination with standard matter source is an LTB-LTB model (in addition to the Bianchi-Bianchi models previously studied in Refs. [30, 79]), subject to some constraints. Combinations like FLRW-Lemaître and FLRW-Bianchi I all require either inhomogeneous or anisotropic sources. We also investigated the question of whether having linear perturbations in just one metric is theoretically consistent. We found, not unexpectedly, that there are no consistent cases except for purely de Sitter backgrounds.

Whether any of the surviving combinations give rise to viable cosmological models at both the background and perturbative levels is, however, entirely to be seen.

\section{ACKNOWLEDGMENTS}

We thank Frank Könnig and Miguel Zumalacárregui for helpful discussions. We also acknowledge support from DFG through the project TRR33 "The Dark Universe." 


\section{Appendix A: Impossibility of mapping Lemaître to FLRW in bigravity}

In this appendix we prove that it is impossible to map the case where the physical metric $g_{\mu \nu}$ is FLRW and the reference metric $f_{\mu \nu}$ has a lapse and scale factor depending on both $r$ and $t$ to a combination where both metrics are of an FRLW form.

We start with the metrics in terms of the coordinates $r$ and $t$,

$$
\begin{aligned}
& g_{\mu \nu} d x^{\mu} d x^{\nu}=-d t^{2}+a^{2}(t) d{\overrightarrow{x_{g}}}^{2}, \\
& f_{\mu \nu} d x^{\mu} d x^{\nu}=-X^{2}(t, r) d t^{2}+b^{2}(t, r) d{\overrightarrow{x_{f}}}^{2},
\end{aligned}
$$

where

$$
\begin{aligned}
d{\overrightarrow{x_{g}}}^{2} & =\frac{d r^{2}}{1-k_{g} r^{2}}+r^{2} d \Omega^{2}, \\
d{\overrightarrow{x_{f}}}^{2} & =\frac{d r^{2}}{1-k_{f} r^{2}}+r^{2} d \Omega^{2},
\end{aligned}
$$

and $d \Omega^{2}=d \theta^{2}+\sin ^{2}(\theta) d \phi^{2}$. We now want to see if it is possible under any conditions to rewrite the metrics in an FLRW form when we transform the coordinates $r$ and $t$ to some new coordinates $\tilde{r}$ and $\tilde{t}$. We assume the new and old coordinates to be related as

$$
\begin{aligned}
t & =T(\tilde{t}, \tilde{r}), \\
r & =R(\tilde{t}, \tilde{r}) .
\end{aligned}
$$

Under these transformations the $g$-metric scale factor $a$ and the $f$-metric scale factor and lapse, $b$ and $X$, should transform as

$$
\begin{aligned}
a(t) & \rightarrow \tilde{a}(\tilde{t}, \tilde{r}), \\
X(t, r) & \rightarrow \tilde{X}(\tilde{t}, \tilde{r}), \\
b(t, r) & \rightarrow \tilde{b}(\tilde{t}, \tilde{r}) .
\end{aligned}
$$

In order to know how the line elements (A3) and (A4) look under the coordinate transformations, we should first see how $d t^{2}$ and $d r^{2}$ transform. We have

$$
\begin{aligned}
& d t^{2}=\dot{T}^{2} d \tilde{t}^{2}+2 T^{\prime} \dot{T} d \tilde{t} d \tilde{r}+T^{\prime 2} d \tilde{r}^{2}, \\
& d r^{2}=\dot{R}^{2} d \tilde{t}^{2}+2 R^{\prime} \dot{R} d \tilde{t} d \tilde{r}+R^{\prime 2} d \tilde{r}^{2},
\end{aligned}
$$

where an overdot denotes a derivative with respect to $\tilde{t}$ and a prime denotes a derivative with respect to $\tilde{r}$. Using all these relations, the transformed line elements for the metrics read

$$
\begin{aligned}
& d \tilde{s}_{g}^{2}=-\left(\dot{T}^{2}-\frac{\tilde{a}^{2}}{1-k_{g} R^{2}} \dot{R}^{2}\right) d \tilde{t}^{2}+2\left(\frac{\tilde{a}^{2}}{1-k_{R} R^{2}} R^{\prime} \dot{R}-T^{\prime} \dot{T}\right) d \tilde{t} d \tilde{r}+\left(\frac{\tilde{a}^{2}}{1-k_{g} R^{2}} R^{\prime 2}-R^{\prime 2}\right) d \tilde{r}^{2}+\tilde{a}^{2} R^{2} d \Omega^{2}, \quad(\mathrm{~A} 12) \\
& d \tilde{s}_{f}^{2}=-\left(\tilde{X}^{2} \dot{T}^{2}-\frac{\tilde{b}^{2}}{1-k_{f} R^{2}} \dot{R}^{2}\right) d \tilde{t}^{2}+2\left(\frac{\tilde{b}^{2}}{1-k_{f} R^{2}} R^{\prime} \dot{R}-\tilde{X}^{2} T^{\prime} \dot{T}\right) d \tilde{t} d \tilde{r}+\left(\frac{\tilde{b}^{2}}{1-k_{f} R^{2}} R^{\prime 2}-\tilde{X}^{2} T^{\prime 2}\right) d \tilde{r}^{2}+\tilde{b}^{2} R^{2} d \Omega^{2} .
\end{aligned}
$$

Now in order to have both metrics in an FLRW form, we need to set the following constraints on our transformed metric components. From Eq. (A12) we obtain

$$
\begin{aligned}
\dot{T}^{2}-\frac{\tilde{a}^{2}}{1-k_{g} R^{2}} \dot{R}^{2} & =A^{2}(\tilde{t}), \\
\frac{\tilde{a}^{2}}{1-k_{g} R^{2}} R^{\prime} \dot{R}-T^{\prime} \dot{T} & =0, \\
\frac{\tilde{a}^{2}}{1-k_{g} R^{2}} R^{\prime 2}-T^{\prime 2} & =\frac{B^{2}(\tilde{t})}{1-\tilde{k}_{g} \tilde{r}^{2}}, \\
\tilde{a}^{2} R^{2} & =B^{2}(\tilde{t}) \tilde{r}^{2},
\end{aligned}
$$


and from Eq. A13 we find

$$
\begin{aligned}
\tilde{X}^{2} \dot{T}^{2}-\frac{\tilde{b}^{2}}{1-k_{f} R^{2}} \dot{R}^{2} & =C^{2}(\tilde{t}), \\
\frac{\tilde{b}^{2}}{1-k_{f} R^{2}} R^{\prime} \dot{R}-\tilde{X}^{2} T^{\prime} \dot{T} & =0, \\
\frac{\tilde{b}^{2}}{1-k_{f} R^{2}} R^{\prime 2}-\tilde{X}^{2} T^{\prime 2} & =\frac{D^{2}(\tilde{t})}{1-\tilde{k}_{f} \tilde{r}^{2}}, \\
\tilde{b}^{2} R^{2} & =D^{2}(\tilde{t}) \tilde{r}^{2},
\end{aligned}
$$

where $A, B, C$, and $D$ are arbitrary functions of only $\tilde{t}$. Now from Eqs. (A17) and (A21), we get

$$
\frac{\tilde{a}^{2}}{\tilde{b}^{2}}=\frac{B^{2}(\tilde{t})}{D^{2}(\tilde{t})}
$$

and from Eqs. (A15) and , w19)

$$
\frac{\widetilde{b}^{2}}{\left(1-k_{f} R^{2}\right) \widetilde{X}^{2}}=\frac{\widetilde{a}^{2}}{1-k_{g} R^{2}} .
$$

Now using the condition (A23) and Eqs. (A14) and (A18), we find

$$
A^{2}(\tilde{t})=\frac{C^{2}(\tilde{t})}{\tilde{X}^{2}} .
$$

This tells us that $\tilde{X}$ must be a function only of $\tilde{t}$. Combining this with Eqs. A22 and A23), we immediately see that $R$ must also be a function only of $\tilde{t}$, and as a result $R^{\prime}=0$. Taking this into account, Eq. A19) implies that

$$
T^{\prime} \dot{T}=0
$$

Let us first discuss the option where $\dot{T}=0$. In this case Eq. A14 tells us that $\tilde{a}$ must be a function only of $\tilde{t}$. Given that $\tilde{a}$ and $\tilde{R}$ are both functions only of $\tilde{t}$, Eq. (A17) immediately gives us a contradiction since the left-hand side is a function only of $\tilde{t}$, while the right-hand side is a function of both $\tilde{t}$ and $\tilde{r}$. For the second option where we assume $T^{\prime}=0$, Eqs. (A16) and (A20) imply that $D(\tilde{t})=B(\tilde{t})=0$, which of course means that we cannot have FRLW metrics under any coordinate transformations.

[1] J. Martin, Comptes Rendus Physique 13, 566 (2012), arXiv:1205.3365 [astro-ph.CO]

[2] L. Amendola and S. Tsujikawa, Dark Energy: Theory and Observations (2010).

[3] T. Clifton, P. G. Ferreira, A. Padilla, and C. Skordis, Phys.Rept. 513, 1 (2012), arXiv:1106.2476 [astro-ph.CO],

[4] M. Fierz and W. Pauli, Proc.Roy.Soc.Lond. A173, 211 (1939)

[5] D. Boulware and S. Deser, Phys.Rev. D6, 3368 (1972).

[6] C. de Rham and G. Gabadadze, Phys.Rev. D82, 044020 (2010), arXiv:1007.0443 [hep-th]

[7] C. de Rham, G. Gabadadze, and A. J. Tolley, Phys.Rev.Lett. 106, 231101 (2011) arXiv:1011.1232 [hep-th].

[8] C. de Rham, G. Gabadadze, and A. J. Tolley, Phys.Lett. B711, 190 (2012) arXiv:1107.3820 [hep-th].

[9] C. de Rham, G. Gabadadze, and A. J. Tolley, JHEP 1111, 093 (2011), arXiv:1108.4521 [hep-th].

[10] S. Hassan and R. A. Rosen, JHEP 1107, 009 (2011), arXiv:1103.6055 [hep-th]

[11] S. Hassan and R. A. Rosen, Phys.Rev.Lett. 108, 041101 (2012) arXiv:1106.3344 [hep-th].

[12] S. Hassan, R. A. Rosen, and A. Schmidt-May, JHEP 1202, 026 (2012), arXiv:1109.3230 [hep-th]

[13] S. Hassan and R. A. Rosen, JHEP 1202, 126 (2012), arXiv:1109.3515 [hep-th]

[14] S. Hassan and R. A. Rosen, JHEP 1204, 123 (2012), arXiv:1111.2070 [hep-th]

[15] C. de Rham, Living Rev.Rel. 17, 7 (2014), arXiv:1401.4173 [hep-th].

[16] G. D’Amico, C. de Rham, S. Dubovsky, G. Gabadadze, D. Pirtskhalava, et al., Phys.Rev. D84, 124046 (2011), arXiv:1108.5231 [hep-th],

[17] A. Higuchi, Nucl.Phys. B282, 397 (1987).

[18] A. E. Gumrukcuoglu, C. Lin, and S. Mukohyama, JCAP 1111, 030 (2011), arXiv:1109.3845 [hep-th]. 
[19] A. E. Gumrukcuoglu, C. Lin, and S. Mukohyama, JCAP 1203, 006 (2012), arXiv:1111.4107 [hep-th].

[20] B. Vakili and N. Khosravi, Phys.Rev. D85, 083529 (2012), arXiv:1204.1456 [gr-qc].

[21] A. De Felice, A. E. Gümrükçüoğlu, and S. Mukohyama, Phys.Rev.Lett. 109, 171101 (2012), arXiv:1206.2080 [hep-th]

[22] M. Fasiello and A. J. Tolley, JCAP 1211, 035 (2012), arXiv:1206.3852 [hep-th].

[23] A. De Felice, A. E. Gümrükçüoğlu, C. Lin, and S. Mukohyama, JCAP 1305, 035 (2013), arXiv:1303.4154 [hep-th].

[24] P. Gratia, W. Hu, and M. Wyman, Phys.Rev. D86, 061504 (2012), arXiv:1205.4241 [hep-th].

[25] A. E. Gümrükçüoğlu, C. Lin, and S. Mukohyama, Phys.Lett. B717, 295 (2012) arXiv:1206.2723 [hep-th].

[26] M. S. Volkov, Phys.Rev. D86, 061502 (2012), arXiv:1205.5713 [hep-th]

[27] M. S. Volkov, Phys.Rev. D86, 104022 (2012) arXiv:1207.3723 [hep-th]

[28] A. De Felice, A. E. Gümrükçüoğlu, C. Lin, and S. Mukohyama, Class.Quant.Grav. 30, 184004 (2013), arXiv:1304.0484 [hep-th].

[29] T. Q. Do and W. Kao, Phys.Rev. D88, 063006 (2013).

[30] W. . Kao and I. C. Lin, Phys.Rev. D90, 063003 (2014).

[31] C. de Rham, M. Fasiello, and A. J. Tolley, (2014), arXiv:1410.0960 [hep-th].

[32] G. D’Amico, G. Gabadadze, L. Hui, and D. Pirtskhalava, Phys.Rev. D87, 064037 (2013), arXiv:1206.4253 [hep-th].

[33] Q.-G. Huang, Y.-S. Piao, and S.-Y. Zhou, Phys.Rev. D86, 124014 (2012) arXiv:1206.5678 [hep-th].

[34] M. Jaccard, M. Maggiore, and E. Mitsou, Phys.Rev. D88, 044033 (2013), arXiv:1305.3034 [hep-th]].

[35] S. Foffa, M. Maggiore, and E. Mitsou, Int.J.Mod.Phys. A29, 1450116 (2014), arXiv:1311.3435 [hep-th].

[36] Y. Dirian, S. Foffa, N. Khosravi, M. Kunz, and M. Maggiore, JCAP 1406, 033 (2014), arXiv:1403.6068 [astro-ph.CO].

[37] A. Conroy, T. Koivisto, A. Mazumdar, and A. Teimouri, Class.Quant.Grav. 32, 015024 (2015), arXiv:1406.4998 [hep-th].

[38] D. Comelli, F. Nesti, and L. Pilo, Phys.Rev. D87, 124021 (2013) arXiv:1302.4447 [hep-th]

[39] D. Comelli, F. Nesti, and L. Pilo, JCAP 1405, 036 (2014), arXiv:1307.8329 [hep-th]

[40] C. de Rham, L. Heisenberg, and R. H. Ribeiro, (2014), arXiv:1408.1678 [hep-th]

[41] C. de Rham, L. Heisenberg, and R. H. Ribeiro, (2014), arXiv:1409.3834 [hep-th]

[42] A. E. Gümrükçüoğlu, L. Heisenberg, and S. Mukohyama, (2014), arXiv:1409.7260 [hep-th].

[43] A. R. Solomon, J. Enander, Y. Akrami, T. S. Koivisto, F. Könnig, et al., (2014), arXiv:1409.8300 [astro-ph.CO]

[44] Y. Yamashita, A. De Felice, and T. Tanaka, (2014), arXiv:1408.0487 [hep-th]

[45] J. Noller and S. Melville, (2014), arXiv:1408.5131 [hep-th]

[46] S. Hassan, M. Kocic, and A. Schmidt-May, (2014), arXiv:1409.1909 [hep-th]

[47] V. O. Soloviev, (2014), arXiv:1410.0048 [hep-th]

[48] L. Heisenberg, (2014), arXiv:1410.4239 [hep-th]

[49] Y. Akrami, T. S. Koivisto, D. F. Mota, and M. Sandstad, JCAP 1310, 046 (2013), arXiv:1306.0004 [hep-th]

[50] Y. Akrami, T. S. Koivisto, and A. R. Solomon, Gen.Rel.Grav. 47, 1838 (2014), arXiv:1404.0006 [gr-qc]]

[51] N. Khosravi, N. Rahmanpour, H. R. Sepangi, and S. Shahidi, Phys.Rev. D85, 024049 (2012) |arXiv:1111.5346 [hep-th]

[52] N. Tamanini, E. N. Saridakis, and T. S. Koivisto, JCAP 1402, 015 (2014), arXiv:1307.5984 [hep-th].

[53] M. S. Volkov, JHEP 1201, 035 (2012), arXiv:1110.6153 [hep-th].

[54] M. von Strauss, A. Schmidt-May, J. Enander, E. Mörtsell, and S. Hassan, JCAP 1203, 042 (2012), arXiv:1111.1655 [gr-qc]

[55] D. Comelli, M. Crisostomi, F. Nesti, and L. Pilo, JHEP 1203, 067 (2012) arXiv:1111.1983 [hep-th]

[56] D. Comelli, M. Crisostomi, and L. Pilo, JHEP 1206, 085 (2012), arXiv:1202.1986 [hep-th].

[57] N. Khosravi, H. R. Sepangi, and S. Shahidi, Phys.Rev. D86, 043517 (2012), arXiv:1202.2767 [gr-qc].

[58] M. Berg, I. Buchberger, J. Enander, E. Mörtsell, and S. Sjörs, JCAP 1212, 021 (2012), arXiv:1206.3496 [gr-qc]

[59] Y. Akrami, T. S. Koivisto, and M. Sandstad, JHEP 1303, 099 (2013) arXiv:1209.0457 astro-ph.CO].

[60] Y. Akrami, T. S. Koivisto, and M. Sandstad, (2013), arXiv:1302.5268 [astro-ph.CO].

[61] J. Enander and E. Mörtsell, JHEP 1310, 031 (2013), arXiv:1306.1086 [astro-ph.CO]

[62] M. Fasiello and A. J. Tolley, JCAP 1312, 002 (2013), arXiv:1308.1647 [hep-th].

[63] F. Könnig, A. Patil, and L. Amendola, JCAP 1403, 029 (2014), arXiv:1312.3208 [astro-ph.CO]

[64] F. Könnig and L. Amendola, Phys.Rev. D90, 044030 (2014) arXiv:1402.1988 [astro-ph.CO]

[65] D. Comelli, M. Crisostomi, and L. Pilo, (2014), arXiv:1403.5679 [hep-th].

[66] A. De Felice, A. E. Gümrükçüoğlu, S. Mukohyama, N. Tanahashi, and T. Tanaka, JCAP 1406, 037 (2014), arXiv:1404.0008 [hep-th].

[67] A. R. Solomon, Y. Akrami, and T. S. Koivisto, JCAP 1410, 066 (2014), arXiv:1404.4061 [astro-ph.CO]

[68] F. Könnig, Y. Akrami, L. Amendola, M. Motta, and A. R. Solomon, Phys.Rev. D90, 124014 (2014), arXiv:1407.4331 [astro-ph.CO].

[69] M. Lagos and P. G. Ferreira, (2014), arXiv:1410.0207 [gr-qc]

[70] G. Cusin, R. Durrer, P. Guarato, and M. Motta, (2014), arXiv:1412.5979 [astro-ph.CO].

[71] J. Enander, Y. Akrami, E. Mortsell, M. Renneby, and A. R. Solomon, (2015), arXiv:1501.02140 [astro-ph.CO].

[72] J. Enander, A. R. Solomon, Y. Akrami, and E. Mörtsell, (2014), arXiv:1409.2860 [astro-ph.CO].

[73] A. Schmidt-May, (2014), arXiv:1409.3146 [gr-qc].

[74] D. Comelli, M. Crisostomi, K. Koyama, L. Pilo, and G. Tasinato, (2015), arXiv:1501.00864 [hep-th]

[75] A. E. Gumrukcuoglu, L. Heisenberg, S. Mukohyama, and N. Tanahashi, (2015), arXiv:1501.02790 [hep-th]

[76] F. Könnig, (2015), arXiv:1503.07436 [astro-ph.CO]

[77] L. Amendola, F. Koennig, M. Martinelli, V. Pettorino, and M. Zumalacarregui, (2015), arXiv:1503.02490 [astro-ph.CO]

[78] M. Johnson and A. Terrana, (2015), arXiv:1503.05560 [astro-ph.CO].

[79] K.-i. Maeda and M. S. Volkov, Phys.Rev. D87, 104009 (2013) arXiv:1302.6198 [hep-th]. 
[80] Y. Akrami, S. Hassan, F. Könnig, A. Schmidt-May, and A. R. Solomon, (2015), arXiv:1503.07521 [gr-qc].

[81] K. Bolejko and P. Lasky, Mon.Not.Roy.Astron.Soc. 391, 59 (2008), arXiv:0809.0334 [astro-ph].

[82] K. Bolejko, M.-N. Celerier, and A. Krasinski, Class.Quant.Grav. 28, 164002 (2011), arXiv:1102.1449 [astro-ph.CO].

[83] G. Lemaitre, Gen.Rel.Grav. 29, 641 (1997).

[84] P. D. Lasky and K. Bolejko, Class.Quant.Grav. 27, 035011 (2010), arXiv:1001.1159 [astro-ph.CO].

[85] T. Harko, F. S. N. Lobo, and M. Mak, Galaxies 2, 496 (2014), arXiv:1410.5213 [gr-qc].

[86] B. Saha, Chin.J.Phys. 43, 1035 (2005), arXiv:gr-qc/0412078 [gr-qc],

[87] V. F. Mukhanov, H. Feldman, and R. H. Brandenberger, Phys.Rept. (1992).

[88] M. Lagos, M. Baados, P. G. Ferreira, and S. Garca-Senz, Phys.Rev. D89, 024034 (2014), arXiv:1311.3828 [gr-qc] 